\title{
In vitro and in vivo Effect of Exogenous Farnesol Exposure Against Candida auris
}

\section{Fruzsina Nagy 1,2, Eszter Vitális ${ }^{2,3}$, Ágnes Jakab4 ${ }^{4}$, Andrew M. Borman ${ }^{5}$, Lajos Forgács ${ }^{1,2}$, Zoltán Tóth ${ }^{1,2}$, László Majoros ${ }^{1}$ and Renátó Kovács ${ }^{1,6 *}$}

${ }^{1}$ Department of Medical Microbiology, Faculty of Medicine, University of Debrecen, Debrecen, Hungary, ${ }^{2}$ Doctoral School of Pharmaceutical Sciences, University of Debrecen, Debrecen, Hungary, ${ }^{3}$ Hospital Hygiene Ward, Clinical Centre, University of Debrecen, Debrecen, Hungary, ${ }^{4}$ Department of Molecular Biotechnology and Microbiology, Faculty of Science and Technology, Institute of Biotechnology, University of Debrecen, Debrecen, Hungary, ${ }^{5}$ UK National Mycology Reference Laboratory, Public Health England, Bristol, United Kingdom, ${ }^{6}$ Faculty of Pharmacy, University of Debrecen, Debrecen, Hungary

The spreading of multidrug-resistant Candida auris is considered as an emerging global health threat. The number of effective therapeutic regimens is strongly limited; therefore, development of novel strategies is needed. Farnesol is a quorum-sensing molecule with a potential antifungal and/or adjuvant effect; it may be a promising candidate in alternative treatment against Candida species including $C$. auris. To examine the effect of farnesol on C. auris, we performed experiments focusing on growth, biofilm production ability, production of enzymes related to oxidative stress, triazole susceptibility and virulence. Concentrations ranging from 100 to $300 \mu \mathrm{M}$ farnesol caused a significant growth inhibition against $C$. auris planktonic cells for $24 \mathrm{~h}(p<0.01-0.05)$. Farnesol treatment showed a concentration dependent inhibition in terms of biofilm forming ability of C. auris; however, it did not inhibit significantly the biofilm development at $24 \mathrm{~h}$. Nevertheless, the metabolic activity of adhered farnesol pre-exposed cells $(75 \mu \mathrm{M})$ was significantly diminished at $24 \mathrm{~h}$ depending on farnesol treatment during biofilm formation $(p<0.001-0.05)$. Moreover, $300 \mu \mathrm{M}$ farnesol exerted a marked decrease in metabolic activity against one-day-old biofilms between 2 and $24 \mathrm{~h}$ $(p<0.001)$. Farnesol increased the production of reactive species remarkably, as revealed by $2^{\prime}, 7^{\prime}$-dichlorofluorescein (DCF) assay $\left\{3.96 \pm 0.89\right.$ [nmol DCF $\left(\mathrm{OD}_{640}\right)^{-1}$ ] and $23.54 \pm 4.51$ [nmol DCF $\left(\mathrm{OD}_{640}\right)^{-1}$ ] for untreated cells and farnesol exposed cells, respectively; $p<0.001\}$. This was in line with increased superoxide dismutase level $\left\{85.69 \pm 5.42\right.$ [munit (mg protein) $^{-1}$ ] and 170.11 \pm 17.37 [munit (mg protein) $^{-1}$ ] for untreated cells and farnesol exposed cells, respectively; $p<0.001\}$, but the catalase level remained statistically comparable between treated and untreated cells ( $p$ > 0.05). Concerning virulence-related enzymes, exposure to $75 \mu \mathrm{M}$ farnesol did not influence phospholipase or aspartic proteinase activity $(p>0.05)$. The interaction between fluconazole, itraconazole, voriconazole, posaconazole, isavuconazole and farnesol showed clear synergism ( $\mathrm{FICl}$ ranges from 0.038 to 0.375 ) against one-day-old biofilms. Regarding in vivo experiments, daily $75 \mu \mathrm{M}$ farnesol treatment decreased the 
fungal burden in an immunocompromised murine model of disseminated candidiasis, especially in case of inocula pre-exposed to farnesol $(p<0.01)$. In summary, farnesol shows a promising therapeutic or adjuvant potential in traditional or alternative therapies such as catheter lock therapy.

Keywords: biofilm, oxidative stress, virulence, in vivo, synergy, triazoles, quorum-sensing, therapy

\section{INTRODUCTION}

Candida auris is an emerging fungal pathogen causing outbreaks in healthcare settings with unacceptably high mortality rates ranging from 28 to $78 \%$ depending on the country (JefferySmith et al., 2017; Eyre et al., 2018). To date, 39 countries have reported C. auris associated infections (Jeffery-Smith et al., 2017; Eyre et al., 2018; Kean et al., 2020). Based on last published data, the number of confirmed $C$. auris infections were 620 and 988 in Europe and United States of America, respectively (European Centre for Disease Prevention and Control, 2018; Centers for Disease Control and Prevention, 2019). Nosocomial C. auris outbreaks were reported from several countries including India, South Africa, Venezuela, Pakistan, and the United States (Lockhart et al., 2017; Vallabhaneni et al., 2017; Belkin et al., 2018). Previously, genetic analyses revealed more genetically unrelated clonal populations across three different continents. These clades are commonly classified as South African, South Asian, East Asian, and South American clades (Lockhart et al., 2017). In addition, a recent study described a fifth $C$. auris clade in Iran from patient who never traveled outside that country (Abastabar et al., 2019; Chow et al., 2019).

Over $90 \%$ of clinical isolates are resistant to fluconazole whereas resistance to newer triazoles is variable (Dudiuk et al., 2019; Romera et al., 2019). The ratio of strains resistant to amphotericin $\mathrm{B}$ ranges from 8 to $50 \%$, while echinocandin resistance remains infrequent (2 to 8\%) (Dudiuk et al., 2019). Alarmingly, isolates of $C$. auris with resistance to all three major antifungal classes have been reported in multiple countries including the United States (Ostrowsky et al., 2020). These multidrug-resistant strains may remain susceptible to nystatin and terbinafine (Sarma and Upadhyay, 2017). C. auris biology have been extensively covered in recent papers (Rossato and Colombo, 2018; Casadevall et al., 2019), however, the data about potential alternative treatment strategies remain scarce (Wall et al., 2018); therefore, there is an urgent need for the development of new antifungal therapies. In addition, multidrugresistance is significantly more frequently reported in the case of C. auris biofilms (Kean and Ramage, 2019). Thus, although the capacity to form biofilms is strain dependent in $C$. auris, they frequently pose a remarkable therapeutic challenge, especially because $C$. auris biofilms also have a considerable virulence capacity (Kean and Ramage, 2019). Since data collected with Candida albicans biofilms cannot be extrapolated to C. auris directly, such studies are urgently needed to meet this novel challenge (Kean and Ramage, 2019).

Farnesol is a fungal quorum-sensing molecule that inhibits yeast-to-hyphae transition and promotes reverse morphogenesis in C. albicans (Hornby et al., 2001). Based on recent studies, farnesol acts synergistically with several antifungal agents against C. albicans, Candida glabrata, Candida tropicalis as well as against Candida parapsilosis planktonic cells and/or biofilms (Katragkou et al., 2015; Kovács et al., 2016; Monteiro et al., 2017; Agustín et al., 2019), thus it has been proposed as a potential adjuvant therapeutic agent. In addition, its therapeutic potential has already been confirmed against $C$. albicans in murine models of mucosal infection (Hisajima et al., 2008; Bozó et al., 2016). Although farnesol is not beneficial in systemic infections caused by C. albicans (Navarathna et al., 2007), those data cannot necessarily be extrapolated to non-albicans species including C. auris (Semreen et al., 2019).

This study examines the effect of farnesol exposure on growth, biofilm production, oxidative stress-related enzyme production, triazole susceptibility and virulence of C. auris, in order to explore the background of the previously observed antifungal effect.

\section{MATERIALS AND METHODS}

\section{Organisms}

Three C. auris isolates (isolates 10, 12, and 27) obtained from National Mycology Reference Laboratory, United Kingdom were used together with the SC5314 C. albicans reference strain. All three C. auris strains derived from the South Asian/Indian lineage (Borman et al., 2017). All C. auris isolates tested showed nonaggregating phenotype, which exhibit comparable pathogenicity to that of C. albicans (Borman et al., 2016).

\section{Toxicity Experiments}

Ten $\mu \mathrm{M}, 50 \mu \mathrm{M}, 150 \mu \mathrm{M}$, and $300 \mu \mathrm{M}$ farnesol were evaluated in terms of toxicity to the Caco-2 cell line using a 3-(4,5-dimethyl2-thiazolyl)-2,5-diphenyl-2H-tetrazolium bromide (MTT) assay (Sigma, Budapest, Hungary) (Berridge et al., 2005). No toxicity was observed with any concentration of farnesol.

\section{Growth Related Experiments for Planktonic Cells}

The effect of pre-exposure and continuous farnesol treatment on C. auris and C. albicans planktonic cells was tested in RPMI-1640 (with L-glutamine and without bicarbonate, $\mathrm{pH} 7.0$ with MOPS; Sigma, Budapest, Hungary) in two experimental settings: (i) effect of various farnesol concentrations against planktonic cells, (ii) effect of various farnesol concentrations against planktonic cells pre-exposed with farnesol $(75 \mu \mathrm{M})$ for 24 -h. Seventy-five $\mu \mathrm{M}$ farnesol was chosen as pre-exposure concentration because it corresponds to approximately double 
the amount of physiological farnesol production of C. albicans (Weber et al., 2008).

Farnesol was obtained as $3 \mathrm{M}$ stock solution, which was diluted to a $30 \mathrm{mM}$ working stock solution in $100 \%$ methanol. The working concentrations of farnesol were prepared in RPMI-1640 medium. Drug-free control was supplemented with $1 \%$ (vol/vol) methanol (Bozó et al., 2016; Kovács et al., 2016; Nagy et al., 2019). Farnesol concentrations tested were 10, 50, 100, and $300 \mu \mathrm{M}$ in all experiments.

Living cell number of planktonic cells was determined using time-kill experiments (Kovács et al., 2014, 2017). Briefly, samples $(100 \mu \mathrm{L})$ were removed at $0,2,4,6,8,10,12$, and $24 \mathrm{~h}$, serially diluted tenfold, plated $(4 \times 30 \mu \mathrm{L})$ onto Sabouraud dextrose agar and incubated at $35^{\circ} \mathrm{C}$ for $48 \mathrm{~h}$. All isolates were tested in three independent experiments and the mean of the three values was used in the analysis. At given time points, one-way ANOVA with Dunnett's post-testing was used to analyze the effect on living cell number exerted by different farnesol concentrations compared to untreated control.

\section{Evaluation of Extracellular \\ Phospholipase and Aspartic Proteinase Activities Exerted by Farnesol Exposure}

Extracellular phospholipase production by farnesol-exposed $(75 \mu \mathrm{M})$ and untreated $C$. auris and C. albicans cells was examined on egg yolk medium [5.85\% (wt/vol) $\mathrm{NaCl}, 0.05 \%$ (wt/vol) $\mathrm{CaCl}_{2}$, and $10 \%$ (vol/vol) sterile egg yolk (Sigma, Budapest, Hungary)]. Aspartic proteinase activity was evaluated on solid medium supplemented with bovine serum albumin $\left[0.02 \%(w t / v o l) \mathrm{MgSO}_{4} \times 7 \mathrm{H}_{2} \mathrm{O}, 0.25 \%(\mathrm{wt} / \mathrm{vol}) \mathrm{K}_{2} \mathrm{HPO}_{4}, 0.5 \%\right.$ (wt/vol) $\mathrm{NaCl}, 0.1 \%$ (wt/vol) yeast extract, $2 \%$ (wt/vol) glucose and $0.25 \%$ (wt/vol) bovine serum albumin (Sigma, Budapest, Hungary) agar medium]. In case of both assay, $5 \mu \mathrm{L}$ suspensions of $1 \times 10^{7}$ cells $/ \mathrm{mL}$ were inoculated onto agar plates as described previously (Kantarcioglu and Yücel, 2002). Colony diameters and precipitation zones $(\mathrm{Pz})$ were measured after 7 days of incubation at $35^{\circ} \mathrm{C}$ (Price et al., 1982). Enzyme activities were measured in three independent experiments for each isolate and are presented as means \pm standard deviations. Statistical analysis of reactive species and enzyme production data were performed by paired Student's $t$-test using GraphPad Prism 6.05 software. The differences between values for treated and control cells were considered significant if the $p$-value was $<0.05$.

\section{Reactive Species Production and Antioxidant Enzyme Activities Exerted by Farnesol Exposure}

Reactive species were measured in the presence or absence of 1-day farnesol (75 $\mu \mathrm{M})$ exposure in RPMI-1640 by a reaction that converts $2^{\prime}, 7^{\prime}$-dichlorofluorescin diacetate to $2^{\prime}, 7^{\prime}$ dichlorofluorescein (DCF) (Sigma, Budapest, Hungary) (Jakab et al., 2015, 2019). The amount of DCF produced is proportional to the quantity of reactive species. Catalase and superoxide dismutase activities were determined as described previously by Jakab et al. (2015, 2019). Reactive species and enzyme activities were measured in three independent experiments for each isolate and are presented as means \pm standard deviations. Statistical comparisons of reactive species and enzyme production data were performed by paired Student's $t$-test using GraphPad Prism 6.05 software. The differences between values for treated and control cells were considered significant if the $p$-value was $<0.05$.

\section{Susceptibility Testing of Planktonic Cells to Azoles and Farnesol}

Antifungal susceptibility of $C$. auris isolates to fluconazole, itraconazole, voriconazole, posaconazole, isavuconazole and to farnesol (all from Sigma, Budapest, Hungary) was tested using the broth microdilution method in RPMI-1640 in line with the CLSI standard M27-A3 guideline (Clinical and Laboratory Standards Institute, 2008). The final concentrations of the drug ranged between 0.5 and $32 \mathrm{mg} / \mathrm{L}, 0.008$ and $0.5 \mathrm{mg} / \mathrm{L}$, and 1.17 and $300 \mu \mathrm{M} \mathrm{mg} / \mathrm{L}$ for fluconazole, other tested azoles and farnesol, respectively. Susceptibility testing for planktonic cells was performed in 96-well microtiter plates at $35^{\circ} \mathrm{C}$ for $24 \mathrm{~h}$. The inoculum was $0.5-2.5 \times 10^{3}$ cells $/ \mathrm{mL}$. Minimum inhibitory concentrations (MICs) were defined as at least 50\% growth reduction compared with untreated control. All isolates were tested in three independent experiments and the median of the three values was used in the analysis.

\section{Biofilm Formation}

Candida isolates were suspended in RPMI-1640 broth at a concentration of $1 \times 10^{6}$ cells $/ \mathrm{mL}$ and aliquots of $100 \mu \mathrm{L}$ were inoculated onto flat-bottom 96-well sterile microtiter plates (TPP, Trasadingen, Switzerland) and then incubated statically at $35^{\circ} \mathrm{C}$ for $24 \mathrm{~h}$ to produce one-day-old biofilms (Pierce et al., 2008; Kovács et al., 2016).

\section{Metabolic Activity Changes of Biofilms Over Time Following Farnesol Exposure}

The effect of pre-exposure and continuous farnesol treatment on C. auris and C. albicans biofilms was tested in three experimental settings: (i) continuous farnesol treatment for 24-h during biofilm formation, (ii) biofilm forming ability of cells pre-exposed with farnesol $(75 \mu \mathrm{M})$ for 24 -h prior to biofilm formation then continuously treated to given farnesol concentrations for 24-h during biofilm development, (iii) effect of farnesol on one-dayold biofilms. Farnesol concentrations tested were 10, 50, 100, and $300 \mu \mathrm{M}$ in all experiments. Metabolic activity of sessile cells was determined at $0,2,4,6,8,10,12$, and $24 \mathrm{~h}$ using XTT-reduction assay (Hawser, 1996; Katragkou et al., 2015). All isolates were tested in three independent experiments and the mean of the three values was used in the analysis. At given time points, one-way ANOVA with Dunnett's post-testing was used to analyze the metabolic activity change exerted by different farnesol concentrations compared to untreated control. The differences between values for treated and control cells were considered significant if the $p$-value was lower than 0.05 .

\section{Susceptibility Testing of Biofilms}

The activity of triazoles and farnesol against one-day-old biofilms was evaluated using the XTT-assay (Hawser, 1996; 
Katragkou et al., 2015; Kovács et al., 2016; Nagy et al., 2019). The concentrations tested in biofilm MIC determination ranged between 8 and $512 \mathrm{mg} / \mathrm{L}, 0.5$ and $32 \mathrm{mg} / \mathrm{L}, 0.125$ and $8 \mathrm{mg} / \mathrm{L}$, and 1.17 and $300 \mu \mathrm{M}$ for fluconazole, voriconazole/itraconazole, posaconazole/isavuconazole and farnesol, respectively. To determine the 24-h biofilm MICs, one-day-old biofilms were first washed three times with $200 \mu \mathrm{L}$ sterile physiological saline. All wells were filled with $100 \mu \mathrm{L}$ of $0.5 \mathrm{~g} / \mathrm{L}$ XTT/1 $\mu \mathrm{M}$ menadione solution. The plates were covered and incubated at $35^{\circ} \mathrm{C}$ for $2 \mathrm{~h}$; afterward, $80 \mu \mathrm{L}$ of the supernatant was removed and transferred into a new sterile 96-well plate to measure the absorbance spectrophotometrically at $492 \mathrm{~nm}$. MICs were defined as the lowest concentration that produced at least $50 \%$ reduction in metabolic activity of fungal biofilms compared to untreated control (Katragkou et al., 2015; Kovács et al., 2016; Nagy et al., 2019). Three independent experiments were performed for all isolates and the median of the three values were presented.

\section{In vitro Interactions Between Farnesol and Azoles for Planktonic Cells and Biofilms}

A fractional inhibitory concentration index (FICI) was used to evaluate drug-drug interactions using a two-dimensional broth microdilution checkerboard assay both for planktonic and sessile cells (Meletiadis et al., 2005; Katragkou et al., 2015; Kovács et al., 2016). In the case of C. albicans, combinations were tested only for biofilms because planktonic isolates are generally susceptible to the tested azoles. The concentration ranges were as described above for MIC determination against planktonic cells and biofilms. The FICI expressed as $\Sigma \mathrm{FIC}=\mathrm{FIC}_{\mathrm{A}}+\mathrm{FIC}_{\mathrm{B}}=$ $\mathrm{MIC}_{\mathrm{A}}$ combination $/ \mathrm{MIC}_{\mathrm{A}}$ alone $+\mathrm{MIC}_{\mathrm{B}}$ combination $/ \mathrm{MIC}_{\mathrm{B}}$ alone, where $\mathrm{MIC}_{\mathrm{A}}$ alone and $\mathrm{MIC}_{\mathrm{B}}$ alone are the MIC values of compounds $\mathrm{A}$ and $\mathrm{B}$ used alone and $\mathrm{MIC}_{\mathrm{A}}$ combination and $\mathrm{MIC}_{\mathrm{B}}{ }^{\text {combination }}$ are the MICs of compounds A and B at the isoeffective combinations, respectively. FICI was defined as the lowest $\Sigma$ FIC (Meletiadis et al., 2005; Katragkou et al., 2015; Kovács et al., 2016). The MIC values of the drugs alone and of all isoeffective combinations were determined as the lowest drug concentrations showing at least $50 \%$ reduction of turbidity for planktonic, or at least $50 \%$ reduction in metabolic activity of biofilm compared to the untreated control cells. The interaction between azoles and farnesol was interpreted as synergistic when FICI was $\leq 0.5$, as indifferent interaction when FICI was between $>0.5$ and 4 and as antagonism when FICI was $>4$ (Meletiadis et al., 2005; Katragkou et al., 2015; Kovács et al., 2016).

\section{In vivo Experiments}

BALB/c immunocompromised female mice (21-23 g) (Charles River) were used to examine the effect of farnesol pre-exposure $(75 \mu \mathrm{M})$ and daily farnesol treatment $(75 \mu \mathrm{M})$ on virulence of C. auris and compared to C. albicans SC5314. The animals were maintained in accordance with the Guidelines for the Care and Use of Laboratory Animals. The experiments were approved by the Animal Care Committee of the University of Debrecen, Debrecen, Hungary (permission no. 12/2014 DEMÁB). Permanent immunosuppression was produced by intraperitoneal administration of $150 \mathrm{mg} / \mathrm{kg}$ cyclophosphamide
4 days prior to infection, $100 \mathrm{mg} / \mathrm{kg}$ cyclophosphamide 1 day prior to infection, $100 \mathrm{mg} / \mathrm{kg}$ cyclophosphamide 2 days postinfection and $100 \mathrm{mg} / \mathrm{kg}$ cyclophosphamide 5 days post-infection (Andes et al., 2010; Kovács et al., 2014). In accordance with our preliminary experiments, mice were challenged intravenously through the lateral tail vein; the infectious doses were $1 \times 10^{7} \mathrm{CFU} / \mathrm{mouse}$ and $8 \times 10^{3} \mathrm{CFU} /$ mouse in $0.2 \mathrm{~mL}$ volume for $C$. auris and C. albicans, respectively. Inoculum density was confirmed by plating serial dilutions on Sabouraud dextrose agar (Kovács et al., 2014). Mice were divided into four groups (10 mice per group); (i) untreated control mice; (ii) inoculation with $24 \mathrm{~h}$-long farnesol pre-exposed $(75 \mu \mathrm{M})$ cells; (iii) there was no farnesol pre-exposure to fungal cells prior to infection, but $75 \mu \mathrm{M}$ daily farnesol treatment (corresponding to approximately $0.4 \mathrm{mg} / \mathrm{kg}$ ) was started from $24 \mathrm{~h}$ post-infection; (iv) $24 \mathrm{~h}$ long farnesol pre-exposure $(75 \mu \mathrm{M})$ to fungal cells prior to infection; afterward, $75 \mu \mathrm{M}$ daily farnesol treatment was started at $24 \mathrm{~h}$ post-infection.

Farnesol treatments were administered intraperitoneally in a volume of $0.5 \mathrm{~mL}$. Control mice were given $0.5 \mathrm{~mL}$ physiological saline intraperitoneally. At 6 days post-infection, mice were euthanized, and their kidneys were removed (Fakhim et al., 2018), weighed and homogenized aseptically. Fungal tissue burden was determined by quantitative culturing. Kidney tissue burden was analyzed using Kruskal-Wallis test with Dunn's post-test (GraphPad Prism 6.05). Significance was defined as $p<0.05$.

\section{Histology}

Kidneys of treated and untreated mice were subjected to histological investigations. Histopathological examination and histochemical staining were performed on routine formalinfixed, paraffin-embedded mouse kidney tissues. Serial 4- $\mu \mathrm{m}-$ thick sections were cut from paraffin blocks, and Periodic acid-Schiff (PAS) staining was performed (Pupim et al., 2017; Kovács et al., 2019).

\section{RESULTS}

\section{Effect of Farnesol on C. auris and C. albicans Planktonic Cell Growth}

Significant decrease was observed in growth rate of $C$. auris for $12 \mathrm{~h}$ in the presence of farnesol concentrations ranges from 50 to $300 \mu \mathrm{M}$ both in case of farnesol unexposed and pre-exposed cells ( $p<0.001-0.05)$ (Figures 1A,B). At 24 h, 100 and $300 \mu \mathrm{M}$ farnesol significantly decreased the viable cell count compared to untreated control in both experimental settings ( $p<0.01-0.001)$ (Figures 1A,B). Surprisingly, neither farnesol pre-exposed nor unexposed C. albicans cells showed significant growth reduction at $24 \mathrm{~h}(p>0.05)$ (Figures 1C,D).

\section{Effects of Farnesol on Extracellular Phospholipase and Proteinase Production of C. auris and C. albicans}

Farnesol treatment did not significantly influence the extracellular proteinase activity of either C. auris or C. albicans. The $P z$ values were $0.83 \pm 0.04$ and $0.82 \pm 0.05$ for $C$. auris 

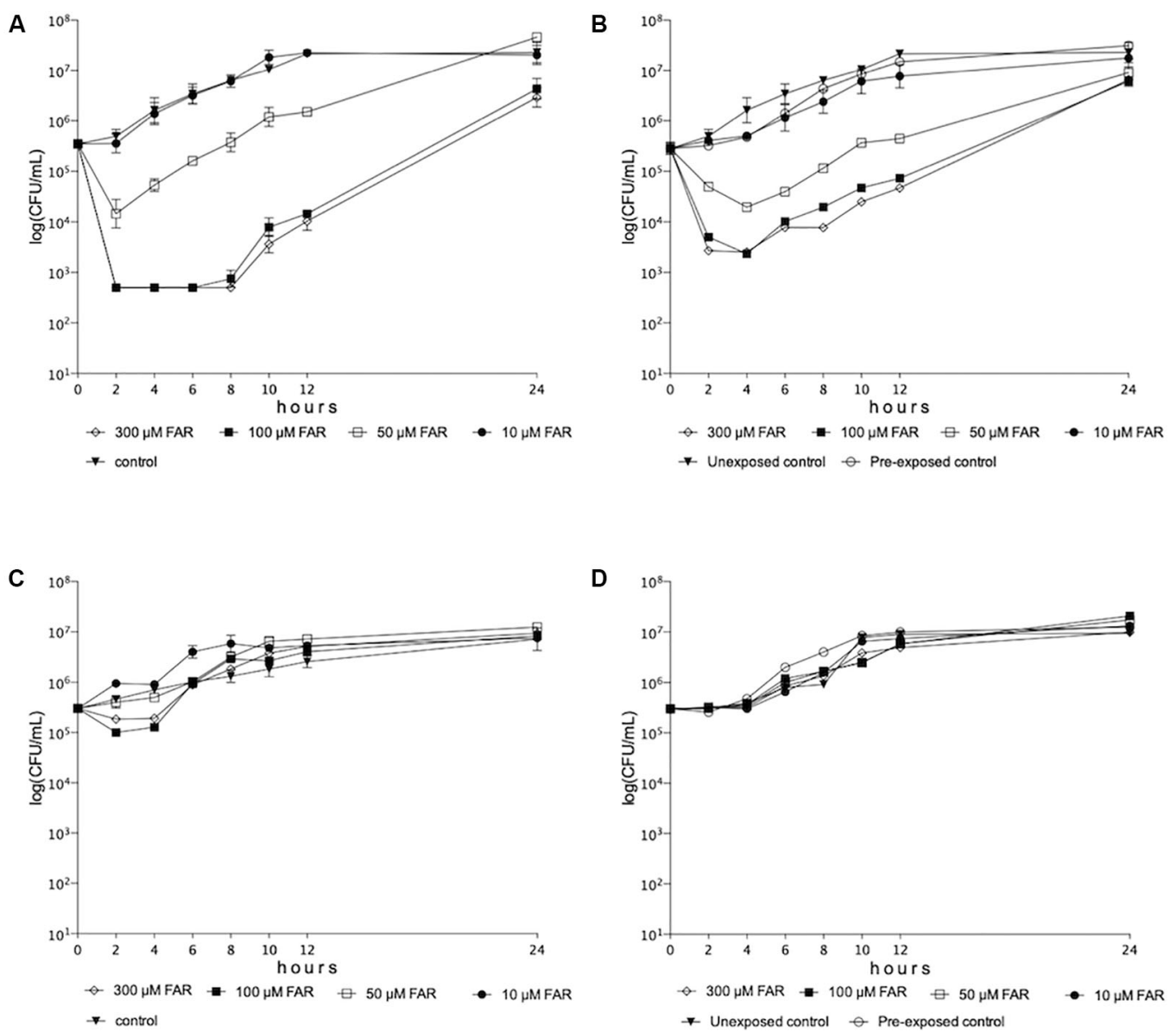

FIGURE 1 | Time-kill curves of farnesol against Candida auris (A,B) and Candida albicans (C,D) isolates in RPMI-1640 for farnesol unexposed (A,C) and farnesol pre-exposed (B,D) cells $(75 \mu \mathrm{M})$, respectively. Each timepoint represents mean \pm SEM (standard error of mean) of cell count derived from isolates.

untreated control and farnesol-exposed cells, respectively $(p>0.05)$, as compared to $0.53 \pm 0.003$ and $0.48 \pm 0.02$ with $C$. albicans untreated control and farnesol-exposed cells, respectively $(p>0.05)$. Farnesol exposure resulted in significantly higher phospholipase activity for $C$. albicans $(P z$ values were $0.48 \pm 0.04$ and $0.42 \pm 0.02$ for untreated control and farnesol-exposed cells, respectively $(p<0.01)$; however, the $P z$ values were statistically comparable in case of $C$. auris $(P z$ values were $0.9 \pm 0.04$ and $0.89 \pm 0.05$ for untreated control and farnesol-exposed cells, respectively $(p>0.05)$.

\section{Farnesol-Induced Oxidative Stress and Stress Response in C. auris and \\ C. albicans}

Farnesol caused a significantly higher reactive species production in $C$. auris compared with untreated control cells as presented in Table $1(p<0.001)$. This farnesol-related higher reactive species level was associated with elevated superoxide dismutase $(p<0.001)$ but statistically comparable catalase activity $(p>0.05)$ (Table 1). Farnesol treatment did not result in significantly higher reactive species production in $C$. albicans $(p>0.05)$, which is in line with the statistically comparable catalase and superoxide dismutase activity between farnesol exposed cells and untreated control $(p>0.05)$ (Table 1).

\section{Effects of Farnesol on Biofilm Forming Ability and One-Day-Old Biofilms of C. auris and C. albicans The Effect of Different Farnesol Concentrations on Biofilm Forming Ability}

All tested farnesol concentrations inhibited the metabolic activity of $C$. auris cells compared to control cells at first $8 \mathrm{~h}(p<0.001-0.05)$; while, statistically comparable metabolic activities were measured at $24 \mathrm{~h}(p>0.05)$ (Figure 2A). In contrast, all tested farnesol concentrations inhibited the metabolic activity of $C$. albicans cells compared to untreated control at $24 \mathrm{~h}$ (Figure 2D).

\section{Biofilm Forming Ability of Cells Pre-exposed With Farnesol for 24-h (75 $\mu \mathrm{M})$ Prior to Biofilm Formation}

Interestingly, we observed statistically significant differences in metabolic activity of $C$. auris cells only at 24 h between 50 and 
TABLE 1 | Farnesol-induced oxidative stress response in Candida auris and Candida albicans.

\begin{tabular}{lcrr}
\hline Oxidative stress related parameter & \multicolumn{2}{c}{ Untreated cultures } & \multicolumn{2}{c}{ Farnesol-exposed cultures } \\
\cline { 2 - 4 } & \multicolumn{1}{c}{ C. auris } & C. albicans & C. auris \\
\hline Catalase $\left[\mathrm{kat}(\mathrm{kg} \text { protein) })^{-1}\right.$ ] & $1.41 \pm 0.03$ & $0.60 \pm 0.07$ & $1.56 \pm 0.09$ \\
SOD [munit (mg protein) ${ }^{-1}$ ] & $85.69 \pm 5.42$ & $78.13 \pm 4.51$ & $170.11 \pm 17.37^{\star \star *}$ \\
DCF [nmol DCF $\left(\mathrm{OD}_{640}\right)^{-1}$ ] & $3.96 \pm 0.89$ & $9.69 \pm 1.01$ & $23.54 \pm 4.51^{\star \star \star}$
\end{tabular}

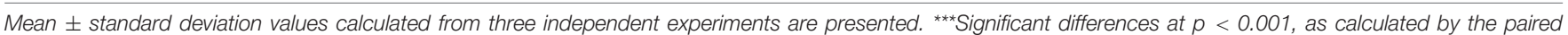
Student's t-test compared to untreated control and farnesol-treated cultures for C. auris. SOD, superoxid dismutase; DCF, 2', $7^{\prime}$-dichlorofluorescein.
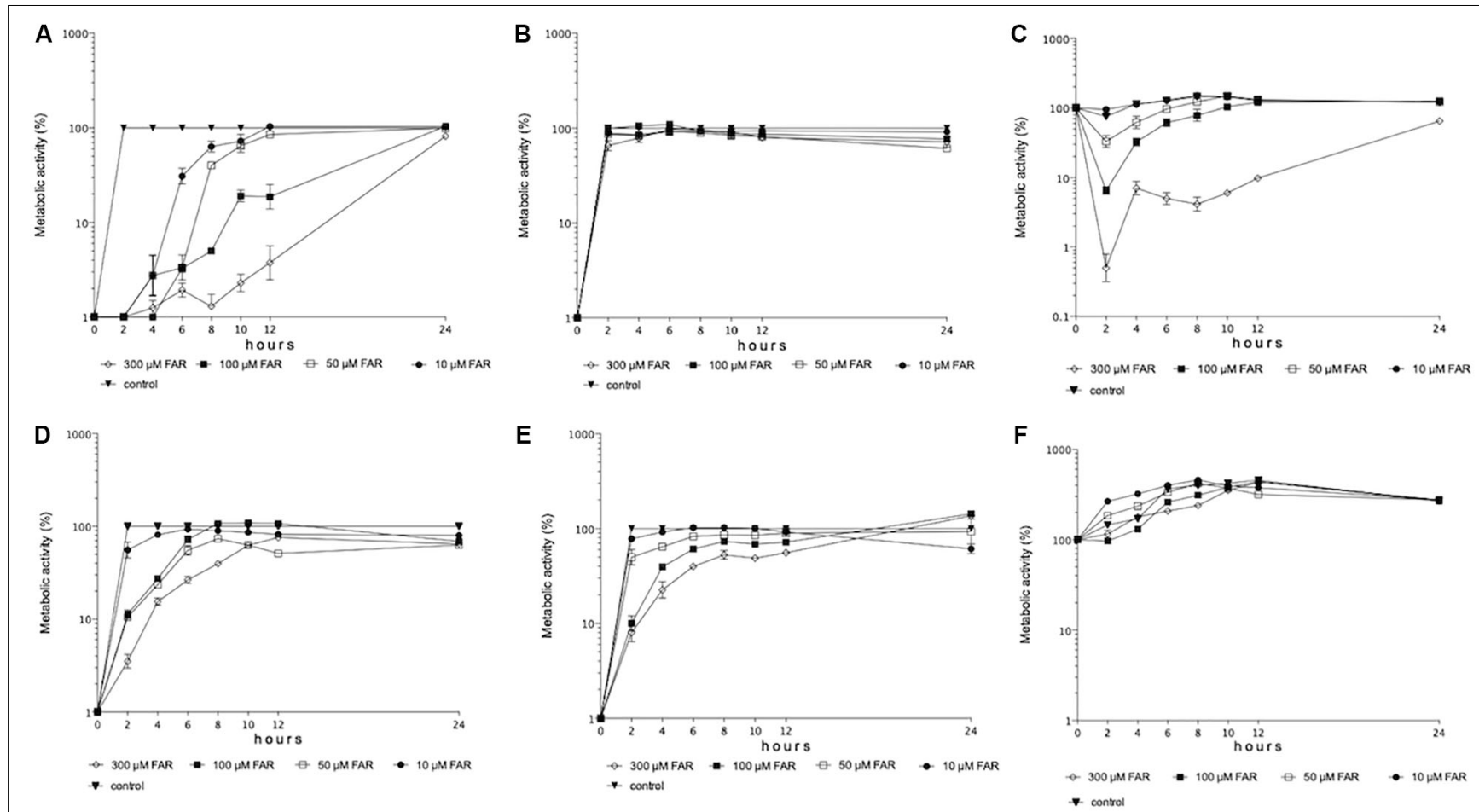

FIGURE 2 | Metabolic activity changes over time in case of biofilm formation in the presence of given farnesol concentrations (10-300 $\mu$ M) for C. auris (A) and C. albicans (D), respectively. Metabolic activity changes over time in case of biofilm formation by farnesol pre-exposed cells (75 $\mu \mathrm{M})$ in the presence of given farnesol concentrations (10-300 $\mu \mathrm{M})$ for C. auris (B) and C. albicans (E), respectively. Metabolic activity changes over time for one-day-old preformed biofilms in the presence of given farnesol concentrations (10-300 $\mu \mathrm{M}$ ) for C. auris (C) and C. albicans (F), respectively. Each time-point represents mean \pm SEM (standard error of mean) of metabolic activity of clinical isolates (three independent experiments per isolate).

$300 \mu \mathrm{M}$ (Figure 2B). In the case of C. albicans, statistically significant differences in metabolic activity between 50 and $300 \mu \mathrm{M}$ were first observed at $8 \mathrm{~h}$ (Figure 2E), but the metabolic activity of cells treated by various concentrations was statistically comparable at $24 \mathrm{~h}$ (Figure 2E).

\section{The Effect of Different Farnesol Concentrations Against One-Day-Old Biofilms}

Between 2 and 24 h, $300 \mu \mathrm{M}$ farnesol produced a potent antibiofilm effect against $C$. auris compared to control (Figure 2C). Interestingly, the low farnesol concentrations $(10-50 \mu \mathrm{M})$ increased the metabolic activity of $C$. albicans biofilms in the first $4 \mathrm{~h}$ (Figure 2F). However, the various farnesol treatments were statistically comparable against $C$. albicans at $24 \mathrm{~h}$ (Figure 2F).

\section{Susceptibility Results for Planktonic Cells and Biofilms}

For C. auris isolates, the planktonic MICs ranged from 4 to $>32 \mathrm{mg} / \mathrm{L}$, from 0.03 to $0.06 \mathrm{mg} / \mathrm{L}$, from 0.008 to $0.015 \mathrm{mg} / \mathrm{L}$, from 0.015 to $0.03 \mathrm{mg} / \mathrm{L}$, and from 0.008 to $0.015 \mathrm{mg} / \mathrm{L}$ for fluconazole, voriconazole, isavuconazole, itraconazole and posaconazole, respectively. The susceptibility to fluconazole of isolate 10 was higher than the tentative fluconazole MIC breakpoint (>32 mg/L) while the other two strains were susceptible to fluconazole (Centers for Disease Control and Prevention, 2020). In the case of planktonic C. albicans SC5314 reference strain, the median MIC values were $0.125 \mathrm{mg} / \mathrm{L}, 0.015 \mathrm{mg} / \mathrm{L}, 0.015 \mathrm{mg} / \mathrm{L}$, $0.125 \mathrm{mg} / \mathrm{L}$, and $0.008 \mathrm{mg} / \mathrm{L}$ for fluconazole, voriconazole, isavuconazole, itraconazole and posaconazole, respectively. 
TABLE 2 | Minimum inhibitory concentration of fluconazole (FLU), voriconazole (VOR), itraconazole (ITRA), posaconazole (POSA) and isavuconazole (ISA) alone and in combination with farnesol (FAR) against Candida auris (10, 12, and 27) and Candida albicans SC5314 biofilms (sMIC).

\begin{tabular}{|c|c|c|c|c|c|c|}
\hline \multirow[t]{3}{*}{ Isolates } & \multicolumn{4}{|c|}{ Median sMIC values } & \multicolumn{2}{|c|}{ Interaction analysis } \\
\hline & \multicolumn{2}{|c|}{ sMIC alone } & \multicolumn{2}{|c|}{ sMIC in combination } & \multirow[t]{2}{*}{ Median FICI } & \multirow[t]{2}{*}{ Type of interaction } \\
\hline & FLU (mg/L) & FAR $(\mu M)$ & FLU (mg/L) & FAR $(\mu \mathrm{M})$ & & \\
\hline 10 & $>512^{a}$ & 300 & 64 & 75 & 0.375 & Synergy \\
\hline 12 & $>512^{\mathrm{a}}$ & 300 & 64 & 75 & 0.35 & Synergy \\
\hline 27 & $>512^{a}$ & 300 & 64 & 75 & 0.375 & Synergy \\
\hline \multirow[t]{2}{*}{ SC5314 } & $>512^{\mathrm{a}}$ & 150 & 64 & 75 & 0.56 & Indifferent \\
\hline & VOR (mg/L) & FAR $(\mu M)$ & VOR (mg/L) & FAR $(\mu M)$ & & \\
\hline 10 & 64 & 150 & 0.5 & 4.69 & 0.093 & Synergy \\
\hline 12 & 64 & 300 & 0.5 & 4.69 & 0.061 & Synergy \\
\hline 27 & 64 & 300 & 0.5 & 9.38 & 0.038 & Synergy \\
\hline \multirow[t]{2}{*}{ SC5314 } & 16 & 150 & 1 & 4.69 & 0.09 & Synergy \\
\hline & ITRA (mg/L) & FAR $(\mu \mathrm{M})$ & ITRA (mg/L) & FAR $(\mu \mathrm{M})$ & & \\
\hline 10 & 16 & 300 & 0.5 & 4.69 & 0.155 & Synergy \\
\hline 12 & 32 & 300 & 0.5 & 9.375 & 0.140 & Synergy \\
\hline 27 & 16 & 300 & 0.5 & 9.375 & 0.123 & Synergy \\
\hline \multirow[t]{2}{*}{ SC5314 } & 8 & 150 & 0.5 & 4.69 & 0.187 & Synergy \\
\hline & POSA (mg/L) & FAR $(\mu \mathrm{M})$ & POSA (mg/L) & FAR $(\mu M)$ & & \\
\hline 10 & 16 & 150 & 0.25 & 2.34 & 0.062 & Synergy \\
\hline 12 & 16 & 150 & 0.25 & 2.34 & 0.062 & Synergy \\
\hline 27 & 16 & 150 & 0.25 & 2.34 & 0.062 & Synergy \\
\hline \multirow[t]{2}{*}{ SC5314 } & 2 & 150 & 0.25 & 4.69 & 0.28 & Synergy \\
\hline & ISA (mg/L) & FAR $(\mu \mathrm{M})$ & ISA (mg/L) & FAR $(\mu \mathrm{M})$ & & \\
\hline 10 & 4 & 300 & 0.125 & 9.38 & 0.091 & Synergy \\
\hline 12 & 8 & 300 & 0.125 & 18.75 & 0.062 & Synergy \\
\hline 27 & 4 & 300 & 0.125 & 9.38 & 0.091 & Synergy \\
\hline SC5314 & 8 & 150 & 0.5 & 4.69 & 0.28 & Synergy \\
\hline
\end{tabular}

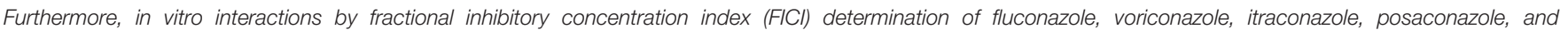

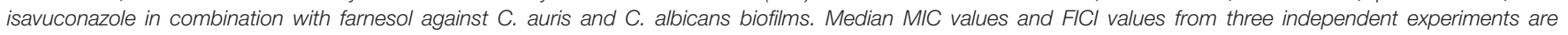
presented. ${ }^{a} \mathrm{MIC}$ is off-scale at $>512 \mathrm{mg} / \mathrm{l}, 1024 \mathrm{mg} / \mathrm{l}$ (one dilution higher than the highest tested concentration) was used for analysis.

In case of biofilms, the median MIC values are shown in Table 2.

\section{Interactions Between Triazoles and Farnesol by FICI}

Only indifferent interactions were detected for planktonic cells of $C$. auris (data not shown). The results of the triazole-farnesol interaction against one-day-old biofilms based on FICI are summarized in Table 2. Antagonism was never observed. Synergy between triazoles and farnesol was observed for all three C. auris isolates when grown in biofilm (FICI ranges from 0.038 to 0.375 ) (Table 2). For the C. albicans SC5314 strain, the interaction pattern observed was very similar to $C$. auris; an indifferent interaction between an azole and farnesol was observed only in case of fluconazole, although, the FICI value calculated was very close to the synergy threshold (Table 2).

\section{In vivo Experiments}

Results of the in vivo experiments are shown in Figures 3, 4 for $C$. auris and C. albicans, respectively. Seventy-five $\mu \mathrm{M}$ farnesol treatment decreased the fungal kidney burden especially when farnesol pre-exposed C. auris cells were used as inoculum (Figure 3). With C. albicans, all experimental settings resulted in statistically comparable kidney fungal burdens compared to untreated control (Figure 4). The histopathology results observed were in line with the fungal burden-related results. C. auris produced single yeast cells and numerous budding yeast cells in untreated control mice. Although, inoculation by farnesol preexposed cells caused large number of aggregates in kidney tissue; the daily farnesol treatment markedly decreased the number of lesions (Figure 3). Both farnesol pre-exposure and daily farnesol treatment caused several extended fungal lesions in kidney tissue in the case of C. albicans infection (Figure 4), where single and 


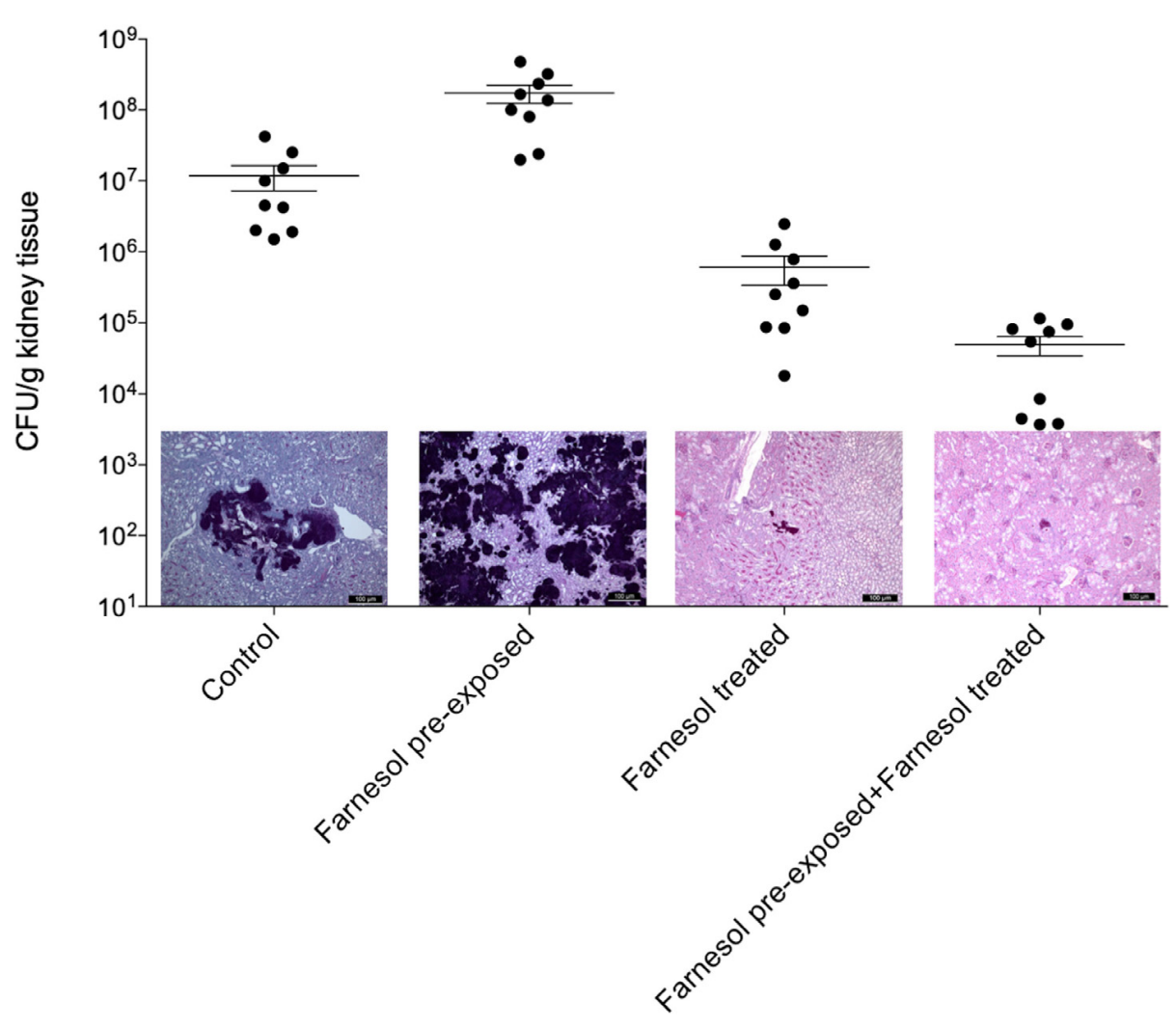

FIGURE 3 | The kidney burden of Candida auris in a systemically infected mouse model. The bars represent the means \pm SEM (standard error of mean) of kidney tissue burdens of BALB/c mice. Significant differences between CFU numbers were determined based on comparison with the untreated controls. Levels of significant differences are indicated $\left({ }^{\star *} p<0.01\right)$. Histological changes in kidney tissue from mice suffering from systemic candidiasis with or without farnesol treatment in the presence or absence of farnesol pre-exposure were examined by Periodic acid-Schiff staining.

budding yeast cells, pseudohyphae and hyphae were observed in all groups (Figure 4).

\section{DISCUSSION}

Only a few classes of antifungal agents are available for the treatment of fungal infections; in addition, the antifungal drug discovery pipeline is slow and challenging, especially in case of the newly emerging difficult-to-treat species such as C. auris (Roemer and Krysan, 2014; Scorzoni et al., 2017). Combination based therapeutic approaches have been proposed as alternatives in recent years to treat the $C$. auris infections. The combination of flucytosine with amphotericin $B$ or micafungin may be relevant for the treatment of $C$. auris infections (Bidaud et al., 2019). Moreover, synergistic interactions were observed between micafungin and voriconazole (Fakhim et al., 2017).

The investigations of alternative/adjuvant treatments focusing on fungal quorum-sensing molecules (e.g., farnesol, tyrosol) have become an intensely researched area in recent years (Mehmood et al., 2019). Several in vitro and in vivo studies were performed to evaluate the antimicrobial effects of farnesol, which revealed that this compound may potentially serve as an alternative or adjuvant drug (Jabra-Rizk et al., 2006; Hisajima et al., 2008; Katragkou et al., 2015; Bozó et al., 2016; Kovács et al., 2016; Nagy et al., 2019). Farnesol has a versatile effect at physiological concentrations, however, the most prominent of these is its ability to influence C. albicans morphology without markedly changing proliferation (Hornby et al., 2001). It is noteworthy that farnesol not only affects C. albicans but has a remarkable inhibitory effect on other non-albicans species and molds especially in supraphysiological concentrations (Jabra-Rizk et al., 2006; Henriques et al., 2007; Rossignol et al., 2007; Weber et al., 2010; Kovács et al., 2016). Our recent study reported that farnesol has a potential antifungal effect against C. auris biofilms (Nagy et al., 2019), nevertheless, the physiological processes underlying the observed antifungal activity of farnesol remain to be elucidated.

Farnesol did not affect the growth rate of planktonic C. albicans; but caused significant reduction in growth rate in the case of $C$. auris. Moreover, farnesol inhibited the metabolic activity of one-day-old biofilms in the first $24 \mathrm{~h}$, a phenomenon clearly absent with C. albicans. The observed farnesol related effect in C. albicans is similar to those reported by Hornby et al. (2001).

Farnesol has been suggested to modulate virulence, since it was shown to affect virulence-associated phospholipase and 


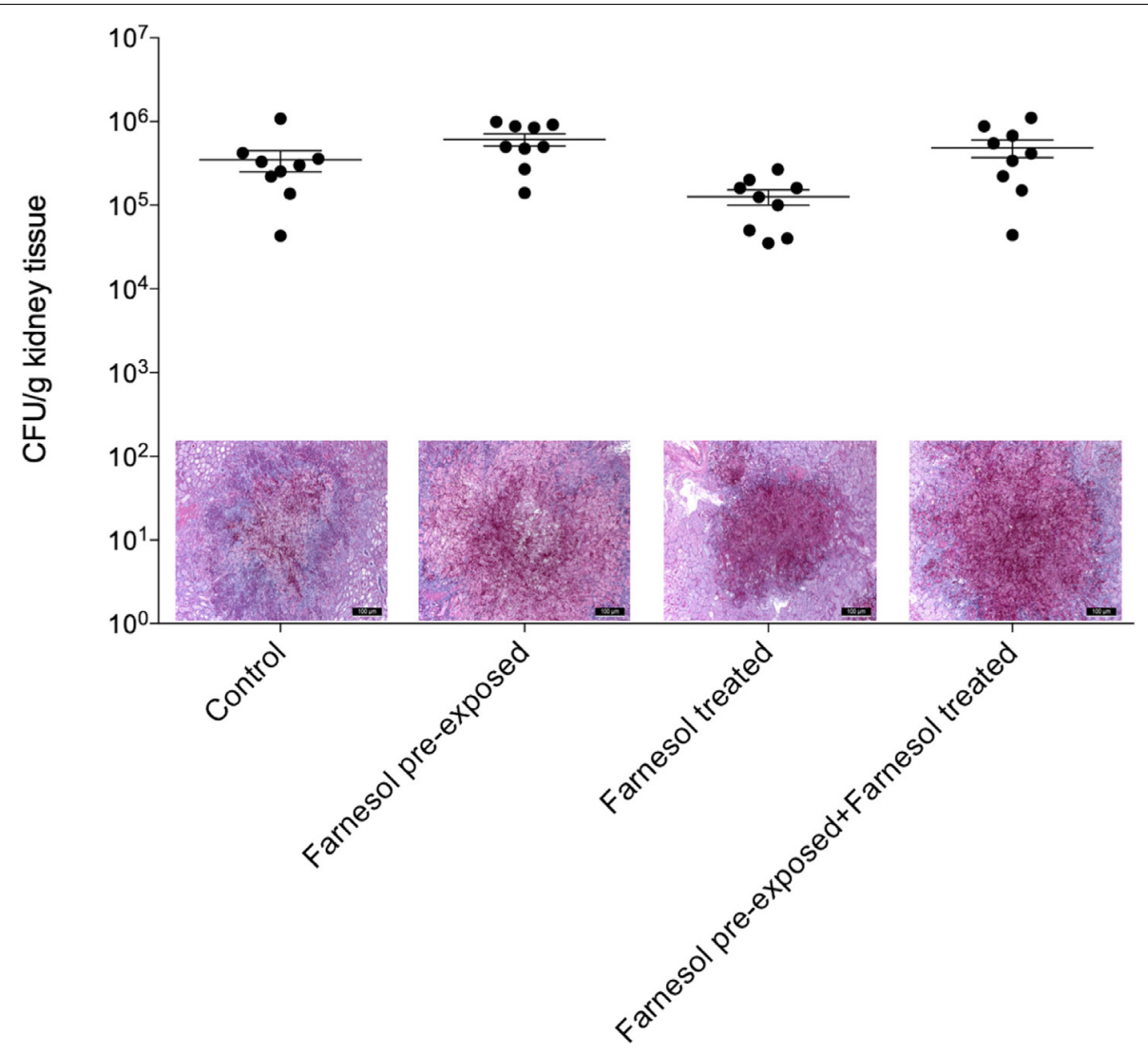

FIGURE 4 | The kidney burden of Candida albicans in a systemically infected mouse model. The bars represent the means \pm SEM (standard error of mean) of kidney tissue burdens of BALB/c mice. Significant differences between CFU numbers were determined based on comparison with the untreated controls. Histological changes in kidney tissue from mice suffering from systemic candidiasis with or without farnesol treatment in the presence or absence of farnesol pre-exposure were examined by Periodic acid-Schiff staining.

aspartyl protease production in C. albicans. In this study, farnesol exposure resulted in significantly higher phospholipase activity for C. albicans, which is line with results reported by Fernandes et al. (2018). However, it did not enhance the production of these enzymes in experiments with $C$. auris.

Farnesol was reported to cause a dose-dependent production of reactive species and could increase resistance to oxidative stress in C. albicans (Davis-Hanna et al., 2008; Deveau et al., 2010), which is concordant with our results. However, farnesol treatment resulted in a significant increase of reactive species production in C. auris, resulting in an elevated level of superoxide dismutase but not catalase, demonstrating that farnesol might not contribute to protection against oxidative stress in C. auris. Such stress-related differences between $C$. albicans and C. auris were also observed previously with other stressor compounds. C. auris was more resistant to hydrogen-peroxide compared to C. albicans; but it was less tolerant to the superoxidegenerating agent menadione and the tert-butyl hydroperoxide, and moreover displayed significantly higher resistance to cationic stress imposed by either sodium chloride or calcium chloride compared to C. albicans (Day et al., 2018).

To date, catheter-associated infections caused by $C$. auris have been reported by several authors, which are attributable to the previously well-documented biofilm-forming ability of this species (Dewaele et al., 2018). Previous studies reported the frequency of central line infections by $C$. auris to be between 11 and 92\% (Lee et al., 2011; Schelenz et al., 2016; Taori et al., 2019). Although sessile communities show significantly higher resistance to the majority of frequently used antifungals compared to planktonic susceptibilities (Kean and Ramage, 2019), the efficacy of such antifungal agents can be enhanced using adjuvants such as farnesol (Nagy et al., 2019). A clear synergy between the tested triazoles and farnesol against $C$. auris biofilms was demonstrated, similarly to the combinations of echinocandins and farnesol (Nagy et al., 2019). Farnesol modulates the expression of genes linked to ergosterol biosynthesis, which may explain the synergy of this compound with triazoles (Yu et al., 2012).

Although the in vitro effect of farnesol is well known especially against C. albicans, its in vivo role remains controversial and raises several questions. Navarathna et al. (2007) showed that exogenous farnesol (20 $\mathrm{mM} /$ mouse) can enhance the pathogenicity of $C$. albicans, increasing the mortality in a murine model of systemic candidiasis. In contrast, Hisajima et al. (2008) observed a farnesol-induced protective effect (at a dose $9 \mu \mathrm{M} /$ mouse) in C. albicans-associated oropharyngeal 
candidiasis. Although Bozó et al. (2016) revealed that farnesol alone is not protective in a murine vulvovaginitis model (150-300 $\mu \mathrm{M} /$ mouse), it did enhance the fluconazole activity against a fluconazole-resistant $C$. albicans isolate. In addition, chitosan nanoparticles containing miconazole and farnesol also inhibited fungal proliferation in a mouse vulvovaginitis model at $\geq 240 \mu \mathrm{M}$ (Fernandes Costa et al., 2019). To the best of our knowledge, there is no reported data concerning the in vivo activity of farnesol against non-albicans Candida species. In this study, daily farnesol treatment decreased the $C$. auris fungal burden in mouse kidneys regardless of previous farnesol exposure of the inoculum. In addition, in the case of inocula pre-exposed to farnesol, the reduction of fungal cell numbers was statistically significant, which is concordant with our in vitro growth-related results. The antifungal activity observed may be explained by the elevated levels of reactive species previously measured in vitro, which could not be detected in equivalent experiments with C. albicans. Furthermore, the amphiphilic properties of farnesol allows for its integration into cell membranes, affecting membrane fluidity and integrity (Bringmann et al., 2000; Funari et al., 2005; Jabra-Rizk et al., 2006; Scheper et al., 2008). Farnesol was shown to affect cellular polarization and membrane permeability in C. parapsilosis and Candida dubliniensis (Jabra-Rizk et al., 2006; Rossignol et al., 2007), which may also explain the observed antifungal effect in our study. However, it is noteworthy that the inoculation of farnesol pre-exposed cells without daily farnesol treatment resulted in a more virulent $C$. auris population and increased fungal burden. The 24-hours-long pre-exposure without further continuous treatment of farnesol may influence the expression of virulence determinants or membrane properties similar to fluconazole pre-treatment, which may explain the virulence enhancer effect reported previously (Navarathna et al., 2005).

\section{CONCLUSION}

In conclusion, our results clearly demonstrate farnesol-related differences in physiology between C. albicans and C. auris. Based on our in vivo studies, farnesol has a remarkable therapeutic potential against $C$. auris; in addition, it reverses the welldocumented resistance to newer triazoles reported for C. auris

\section{REFERENCES}

Abastabar, M., Haghani, I., Ahangarkani, F., Rezai, M. S., Taghizadeh Armaki, M., Roodgari, S., et al. (2019). Candida auris otomycosis in Iran and review of recent literature. Mycoses 62, 101-105. doi: 10.1111/myc.12886

Agustín, M. D. R., Viceconte, F. R., Vela Gurovic, M. S., Costantino, A., and Brugnoni, L. I. (2019). Effect of quorum sensing molecules and natamycin on biofilms of Candida tropicalis and other yeasts isolated from industrial juice filtration membranes. J. Appl. Microbiol. 126, 1808-1820. doi: 10.1111/jam. 14248

Andes, D., Diekema, D. J., Pfaller, M. A., Bohrmuller, J., Marchillo, K., and Lepak, A. (2010). In vivo comparison of the pharmacodynamic targets for echinocandin drugs against Candida species. Antimicrob. Agents Chemother. 54, 2497-2506. doi: 10.1128/AAC.01584-09 biofilms. However, further genome-wide gene expression analysis with $C$. auris is needed in order that each aspect of farnesolrelated effects (e.g., short-term exposure vs. long-term exposure) can be elucidated.

\section{DATA AVAILABILITY STATEMENT}

All datasets generated for this study are included in the article/supplementary material.

\section{ETHICS STATEMENT}

The animal study was reviewed and approved by the Animal Care Committee of the University of Debrecen, Debrecen, Hungary (permission no. 12/2014 DEMÁB).

\section{AUTHOR CONTRIBUTIONS}

RK conceived the ideas, analyzed the data, and wrote the manuscript. FN performed the biofilm forming related tests and susceptibility tests, in vivo tests, and wrote the manuscript. EV performed the growth related experiments. ÁJ performed the oxidative stress related experiments. $\mathrm{AB}$ provided the strains and interpreted the several results. LF performed the histological examinations. ZT performed the biofilm forming related tests, susceptibility tests, and in vivo tests. LM performed the in vivo experiments and analyzed the data.

\section{FUNDING}

RK was supported by the EFOP-3.6.3-VEKOP-16-2017-00009 program. ZT and FN were supported by the UNKP-19-3 New National Excellence Program of the Ministry of Human Capacities.

\section{ACKNOWLEDGMENTS}

The authors thank Dániel Nemes for toxicity experiments.

Belkin, A., Gazit, Z., Keller, N., Ben-Ami, R., Wieder-Finesod, A., Novikov, A. et al. (2018). Candida auris infection leading to nosocomial transmission, Israel, 2017. Emerg. Infect. Dis. 24, 801-804. doi: 10.3201/eid2404.171715

Berridge, M. V., Herst, P. M., and Tan, A. S. (2005). Tetrazolium dyes as tools in cell biology: new insights into their cellular reduction. Biotechnol. Annu. Rev. 11, 127-152. doi: 10.1016/S1387-2656(05)11004-7

Bidaud, A. L., Botterel, F., Chowdhary, A., and Dannaoui, E. (2019). In vitro antifungal combination of flucytosine with amphotericin B, voriconazole, or micafungin against Candida auris shows no antagonism. Antimicrob. Agents Chemother. 63:e1393-19.

Borman, A. M., Szekely, A., and Johnson, E. M. (2016). Comparative pathogenicity of United Kingdom isolates of the emerging pathogen Candida auris and other key pathogenic Candida species. mSphere 1:e189-16. doi: 10.1128/mSphere. 00189-16 
Borman, A. M., Szekely, A., and Johnson, E. M. (2017). Isolates of the emerging pathogen Candia auris present in the UK have several geographic origins. Med. Mycol. 55, 563-567. doi: 10.1093/mmy/myw147

Bozó, A., Domán, M., Majoros, L., Kardos, G., Varga, I., and Kovács, R. (2016). The in vitro and in vivo efficacy of fluconazole in combination with farnesol against Candida albicans isolates using a murine vulvovaginitis model. J. Microbiol. 54, 753-760. doi: 10.1007/s12275-016-6298-y

Bringmann, A., Skatchkov, S. N., Faude, F., Enzmann, V., and Reichenbach, A. (2000). Farnesol modulates membrane currents in human retinal glial cells. J. Neurosci. Res. 62, 396-402. doi: 10.1002/1097-4547(20001101)62:3<396::AIDJNR10>3.0.CO;2-E

Casadevall, A., Kontoyiannis, D. P., and Robert, V. (2019). On the emergence of Candida auris: climate change, azoles, swamps, and birds. mBio 10:e1397-19. doi: 10.1128/mBio.01397-19

Centers for Disease Control and Prevention (2019). Tracking Candida auris. Avialable at: https://www.cdc.gov/fungal/candida-auris/tracking-c-auris.html. (accessed December 31, 2019).

Centers for Disease Control and Prevention (2020). Antifungal Susceptibility Testing and Interpretation. Available online at: https://www.cdc.gov/fungal/ candida-auris/c-auris-antifungal.html (accessed January 2, 2020).

Chow, N. A., de Groot, T., Badali, H., Abastabar, M., Chiller, T. M., and Meis, J. F. (2019). Potential fifth clade of Candida auris, Iran, 2018. Emerg. Infect. Dis. 25, 1780-1781. doi: 10.3201/eid2509.190686

Clinical and Laboratory Standards Institute (2008). Reference Method for Broth Dilution Antifungal Susceptibility Testing of Yeasts. Approved Standard. $3 r d$ ed. M27-A3. Wayne, PA: CLSI.

Davis-Hanna, A., Piispanen, A. E., Stateva, L. I., and Hogan, D. A. (2008). Farnesol and dodecanol effects on the Candida albicans Ras1-cAMP signalling pathway and the regulation of morphogenesis. Mol. Microbiol. 67, 47-62. doi: 10.1111/j. 1365-2958.2007.06013.x

Day, A. M., McNiff, M. M., da Silva, Dantas, A., Gow, N. A. R., and Quinn, J. (2018). Hogl regulates stress tolerance and virulence in the emerging fungal pathogen Candida auris. $m$ Sphere 3:e0506-18. doi: 10.1128/mSphere.00506-18

Deveau, A., Piispanen, A. E., Jackson, A. A., and Hogan, D. A. (2010). Farnesol induces hydrogen peroxide resistance in Candida albicans yeast by inhibiting the Ras-cyclic AMP signaling pathway. Eukaryot. Cell 9, 569-577. doi: 10.1128/ EC.00321-09

Dewaele, K., Frans, J., Smismans, A., Ho, E., Tollens, T., and Lagrou, K. (2018). First case of Candida auris infection in Belgium in a surgical patient from Kuwait. Acta Clin. Belg. [Epub ahead of print].

Dudiuk, C., Berrio, I., Leonardelli, F., Morales-Lopez, S., Theill, L., Macedo, D., et al. (2019). Antifungal activity and killing kinetics of anidulafungin, caspofungin and amphotericin B against Candida auris. J. Antimicrob. Chemother. 74, 2295-2302. doi: 10.1093/jac/dkz178

European Centre for Disease Prevention and Control (2018). Candida auris in Healthcare Settings Europe. Available online at: https://ecdc.europa.eu/sites/ portal/files/documents/RRA-Candida-auris-European-Union-countries.pdf. (accessed November 5, 2018).

Eyre, D. W., Sheppard, A. E., Madder, H., Morales-Lopez, S., Theill, L., Macedo, D., et al. (2018). A Candida auris outbreak and its control in an intensive care setting. N. Engl. J. Med. 379, 1322-1331. doi: 10.1056/NEJMoa1714373

Fakhim, H., Chowdhary, A., Prakash, A., Vaezi, A., Dannaoui, E., Meis, J. F., et al. (2017). In Vitro interactions of echinocandins with triazoles against multidrugresistant Candida auris. Antimicrob. Agents Chemother. 61:e01056-17. doi: 10. 1128/AAC.01056-17

Fakhim, H., Vaezi, A., Dannaoui, E., Chowdhary, A., Nasiry, D., Faeli, L., et al. (2018). Comparative virulence of Candida auris with Candida haemulonii, Candida glabrata and Candida albicans in a murine model. Mycoses 61, 377382. doi: $10.1111 /$ myc. 12754

Fernandes, R. A., Monteiro, D. R., Arias, L. S., Fernandes, G. L., Delbem, A. C. B., and Barbosa, D. B. (2018). Virulence factors in Candida albicans and Streptococcus mutans biofilms mediated by farnesol. Indian J. Microbiol. 58, 138-145. doi: 10.1007/s12088-018-0714-4

Fernandes Costa, A., Evangelista Araujo, D., Santos Cabral, M., Teles Brito, I., Borges de Menezes Leite, L., Pereira, M., et al. (2019). Development, characterization, and in vitro-in vivo evaluation of polymeric nanoparticles containing miconazole and farnesol for treatment of vulvovaginal candidiasis. Med. Mycol. 57, 52-62. doi: 10.1093/mmy/myx155
Funari, S. S., Prades, J., Escribá, P. V., and Barceló, F. (2005). Farnesol and geranylgeraniol modulate the structural properties of phosphatidylethanolamine model membranes. Mol. Membr. Biol. 22, 303-311. doi: 10.1080/09687860500135411

Hawser, S. (1996). Adhesion of different Candida spp. to plastic: XTT formazan determinations. J. Med. Vet. Mycol. 34, 407-410. doi: 10.1080/ 02681219680000721

Henriques, M., Martins, M., Azeredo, J., and Oliveira, R. (2007). Effect of farnesol on Candida dubliniensis morphogenesis. Lett. Appl. Microbiol. 44, 199-205. doi: 10.1111/j.1472-765X.2006.02044.X

Hisajima, T., Maruyama, N., Tanabe, Y., Ishibashi, H., Yamada, T., Makimura, K., et al. (2008). Protective effects of farnesol against oral candidiasis in mice. Microbiol. Immunol. 52, 327-333. doi: 10.1111/j.1348-0421.2008.00044.x

Hornby, J. M., Jensen, E. C., Lisec, A. D., Tasto, J. J., Jahnke, B., Shoemaker, R., et al. (2001). Quorum sensing in the dimorphic fungus Candida albicans is mediated by farnesol. Appl. Environ. Microbiol. 67, 2982-2992. doi: 10.1128/AEM.67.7. 2982-2992.2001

Jabra-Rizk, M. A., Shirtliff, M., James, C., and Meiller, T. (2006). Effect of farnesol on Candida dubliniensis biofilm formation and fluconazole resistance. FEMS Yeast Res. 6, 1063-1073. doi: 10.1111/j.1567-1364.2006.00121.x

Jakab, Á., Emri, T., Sipos, L., Kiss, Á., Kovács, R., Dombrádi, V., et al. (2015). Betamethasone augments the antifungal effect of menadione-towards a novel anti-Candida albicans combination therapy. J. Basic Microbiol. 55, 973-981. doi: 10.1002/jobm.201400903

Jakab, Á, Tóth, Z., Nagy, F., Nemes, D., Bácskay, I., Kardos, G., et al. (2019). Physiological and transcriptional responses of Candida parapsilosis to exogenous tyrosol. Appl. Environ. Microbiol. 85:e01388-19. doi: 10.1128/AEM. 01388-19

Jeffery-Smith, A., Taori, S. K., Schelenz, S., Jeffery, K., Johnson, E. M., Borman, A., et al. (2017). Candida auris: a review of the literature. Clin. Microbiol. Rev. 31:e0029-17. doi: 10.1128/CMR.00029-17

Kantarcioglu, A. S., and Yücel, A. (2002). Phospholipase and protease activities in clinical Candida isolates with reference to the sources of strains. Mycoses 45, 160-165. doi: 10.1046/j.1439-0507.2002.00727.x

Katragkou, A., McCarthy, M., Alexander, E. L., Antachopoulos, C., Meletiadis, J., Jabra-Rizk, M. A., et al. (2015). In vitro interactions between farnesol and fluconazole, amphotericin B or micafungin against Candida albicans biofilms. J. Antimicrob. Chemother. 70, 470-478. doi: 10.1093/jac/dku374

Kean, R., Brown, J., Gulmez, D., Ware, A., and Ramage, G. (2020). Candida auris: a decade of understanding of an enigmatic pathogenic yeast. J. Fungi 6:E30. doi: $10.3390 /$ jof6010030

Kean, R., and Ramage, G. (2019). Combined antifungal resistance and biofilm tolerance: the global threat of Candida auris. mSphere 4:e458-19. doi: 10.1128/ mSphere.00458-19

Kovács, R., Bozó, A., Gesztelyi, R., Domán, M., Kardos, G., Nagy, F., et al. (2016). Effect of caspofungin and micafungin in combination with farnesol against Candida parapsilosis biofilms. Int. J. Antimicrob. Agents 47, 304-310. doi: 10. 1016/j.ijantimicag.2016.01.007

Kovács, R., Gesztelyi, R., Berényi, R., Domán, M., Kardos, G., Juhász, B., et al. (2014). Killing rates exerted by caspofungin in 50\% serum and its correlation with in vivo efficacy in a neutropenic murine model against Candida krusei and Candida inconspicua. J. Med. Microbiol. 63(Pt 2), 186-194. doi: 10.1099/jmm.0. 066381-0

Kovács, R., Holzknecht, J., Hargitai, Z., Papp, C., Farkas, A., Borics, A., et al. (2019). In Vivo applicability of Neosartorya fischeri antifungal Protein 2 (NFAP2) in treatment of vulvovaginal candidiasis. Antimicrob. Agents Chemother. 63:e01777-18. doi: 10.1128/AAC.01777-18

Kovács, R., Saleh, Q., Bozó, A., Tóth, Z., Gesztelyi, R., Kardos, T., et al. (2017). Killing activity of micafungin against Candida albicans, C. dubliniensis and Candida africana in the presence of human serum. Mycopathologia 182, 979987. doi: 10.1007/s11046-017-0178-9

Lee, W. G., Shin, J. H., Uh, Y., Kang, M. G., Kim, S. H., Park, K. H., et al. (2011). First three reported cases of nosocomial fungemia caused by Candida auris. J. Clin. Microbiol. 49, 3139-3142. doi: 10.1128/JCM.00319-11

Lockhart, S. R., Etienne, K. A., Vallabhaneni, S., Farooqi, J., Chowdhary, A., Govender, N. P., et al. (2017). Simultaneous emergence of multidrug-resistant Candida auris on 3 continents confirmed by whole-genome sequencing and epidemiological analyses. Clin. Infect. Dis. 64, 134-140. doi: 10.1093/cid/ciw691 
Mehmood, A., Liu, G., Wang, X., Meng, G., Wang, C., and Liu, Y. (2019). Fungal quorum-sensing molecules and inhibitors with potential antifungal activity: a review. Molecules 24:1950. doi: 10.3390/molecules24101950

Meletiadis, J., Verweij, P. E., TeDorsthorst, D. T., Meis, J. F., and Mouton, J. W. (2005). Assessing in vitro combinations of antifungal drugs against yeasts and filamentous fungi: comparison of different drug interaction models. Med. Mycol. 43, 133-152. doi: 10.1080/13693780410001731547

Monteiro, D. R., Arias, L. S., Fernandes, R. A., Deszo da Silva, L. F., de Castilho, M. O. V. F., da Rosa, T. O., et al. (2017). Antifungal activity of tyrosol and farnesol used in combination against Candida species in the planktonic state or forming biofilms. J. Appl. Microbiol. 123, 392-400. doi: 10.1111/jam.13513

Nagy, F., Tóth, Z., Daróczi, L., Székely, A., Borman, A. M., Majoros, L., et al. (2019). Farnesol increases the activity of echinocandins against Candida auris biofilms. Med. Mycol. 2019, 404-407. doi: 10.1093/mmy/myz057

Navarathna, D. H., Hornby, J. M., Hoerrmann, N., Parkhurst, A. M., Duhamel, G. E., and Nickerson, K. W. (2005). Enhanced pathogenicity of Candida albicans pre-treated with subinhibitory concentrations of fluconazole in a mouse model of disseminated candidiasis. J. Antimicrob. Chemother. 56, 11561159. doi: 10.1093/jac/dki383

Navarathna, D. H., Hornby, J. M., Krishnan, N., Parkhurst, A., Duhamel, G. E., and Nickerson, K. W. (2007). Effect of farnesol on a mouse model of systemic candidiasis, determined by use of a DPP3 knockout mutant of Candida albicans. Infect. Immun. 75, 1609-1618. doi: 10.1128/IAI.01182-06

Ostrowsky, B., Greenko, J., Adams, E., Quinn, M., O’Brien, B., Chaturvedi, V., et al. (2020). Candida auris isolates resistant to three classes of antifungal medications - New York, 2019. MMWR Morb. Mortal. Wkly. Rep. 69, 6-9. doi: 10.15585/mmwr.mm6901a2

Pierce, C. G., Uppuluri, P., Tristan, A. R., Wormley, F. L. Jr., Mowat, E., Ramage, G., et al. (2008). A simple and reproducible 96-well plate-based method for the formation of fungal biofilms and its application to antifungal susceptibility testing. Nat. Protoc. 3, 1494-1500. doi: 10.1038/nport.2008.141

Price, M. F., Wilkinson, I. D., and Gentry, L. O. (1982). Plate method for detection of phospholipase activity in Candida albicans. Sabouraudia 20, 7-14. doi: 10. 1080/00362178285380031

Pupim, A. C. E., Campois, T. G., Araújo, E. J. A., Svidizinski, T. I. E., and Felipe, I. (2017). Infection and tissue repair of experimental cutaneous candidiasis in diabetic mice. J. Med. Microbiol. 66, 808-815. doi: 10.1099/jmm.0.000496

Roemer, T., and Krysan, D. J. (2014). Antifungal drug development: challenges, unmet clinical needs, and new approaches. Cold Spring Harb. Perspect. Med. 4:a019703. doi: 10.1101/cshperspect.a019703

Romera, D., Aguilera-Correa, J. J., Gadea, I., Viñuela-Sandoval, L., GarcíaRodríguez, J., and Esteban, J. (2019). Candida auris: a comparison between planktonic and biofilm susceptibility to antifungal drugs. J. Med. Microbiol. 68, 1353-1358. doi: 10.1099/jmm.0.001036

Rossato, L., and Colombo, A. L. (2018). Candida auris: what have we learned about its mechanisms of pathogenicity? Front. Microbiol. 9:3081. doi: 10.3389/fmicb. 2018.03081

Rossignol, T., Logue, M. E., Reynolds, K., Grenon, M., Lowndes, N. F., and Butler, G. (2007). Transcriptional response of Candida parapsilosis following exposure to farnesol. Antimicrob. Agents Chemother. 51, 2304-2312. doi: 10.1128/AAC. 01438.06

Sarma, S., and Upadhyay, S. (2017). Current perspective on emergence, diagnosis and drug resistance in Candida auris. Infect. Drug Resist. 10, 155-165. doi: 10.2147/IDR.S116229
Schelenz, S., Hagen, F., Rhodes, J. L., Abdolrasouli, A., Chowdhary, A., Hall, A., et al. (2016). First hospital outbreak of the globally emerging Candida auris in a European hospital. Antimicrob. Resist. Infect. Control. 5:35. doi: 10.1186/ s13756-016-0132-5

Scheper, M. A., Shirtliff, M. E., Meiller, T. F., Peters, B. M., and Jabra-Rizk, M. A. (2008). Farnesol, a fungal quorum-sensing molecule triggers apoptosis in human oral squamous carcinoma cells. Neoplasia 10, 954-963. doi: 10.1593/ neo.08444

Scorzoni, L., de Paula, E., Silva, A. C., Marcos, C. M., Assato, P. A., de Melo, W. C., et al. (2017). Antifungal therapy: new advances in the understanding and treatment of mycosis. Front. Microbiol. 8:36. doi: 10.3389/fmicb.2017.00036

Semreen, M. H., Soliman, S. S. M., Saeed, B. Q., Alqarihi, A., Uppuluri, P., and Ibrahim, A. S. (2019). Metabolic profiling of Candida auris, a newly-emerging multi-drug resistant Candida species, by GC-MS. Molecules 24:399. doi: 10. 3390/molecules24030399

Taori, S. K., Khonyongwa, K., Hayden, I., Athukorala, G. D. A., Letters, A., Fife, A., et al. (2019). Candida auris outbreak: mortality, interventions and cost of sustaining control. J. Infect. 79, 601-611. doi: 10.1016/j.jinf.2019. 09.007

Vallabhaneni, S., Kallen, A., Tsay, S., Chow, N., Welsh, R., Kerins, J., et al. (2017). Investigation of the first seven reported cases of Candida auris, a globally emerging invasive, multidrug-resistant fungus-United States, May 2013-August 2016. Am. J. Transplant. 17, 296-299. doi: 10.1111/ajt.14121

Wall, G., Chaturvedi, A. K., Wormley, F. L. Jr., Wiederhold, N. P., Patterson, H. P., Patterson, T. F., et al. (2018). Screening a repurposing library for inhibitors of multidrug-resistant Candida auris identifies ebselen as a repositionable candidate for antifungal drug development. Antimicrob. Agents Chemother. 62:e1084-18. doi: 10.1128/AAC.01084- 18

Weber, K., Schulz, B., and Ruhnke, M. (2010). The quorum-sensing molecule E,Efarnesol-its variable secretion and its impact on the growth and metabolism of Candida species. Yeast 27, 727-739. doi: 10.1002/yea.1769

Weber, K., Sohr, R., Schulz, B., Fleischhacker, M., and Ruhnke, M. (2008). Secretion of E,E-farnesol and biofilm formation in eight different Candida species. Antimicrob. Agents Chemother. 52, 1859-1861. doi: 10.1128/AAC.016 46-07

Yu, L. H., Wei, X., Ma, M., Chen, X. J., and Xu, S. B. (2012). Possible inhibitory molecular mechanism of farnesol on the development of fluconazole resistance in Candida albicans biofilm. Antimicrob. Agents Chemother. 56, 770-775. doi: 10.1128/AAC.05290-11

Conflict of Interest: LM received conference travel grants from Cidara, MSD, Astellas and Pfizer.

The remaining authors declare that the research was conducted in the absence of any commercial or financial relationships that could be construed as a potential conflict of interest.

Copyright (c) 2020 Nagy, Vitális, Jakab, Borman, Forgács, Tóth, Majoros and Kovács. This is an open-access article distributed under the terms of the Creative Commons Attribution License (CC BY). The use, distribution or reproduction in other forums is permitted, provided the original author(s) and the copyright owner(s) are credited and that the original publication in this journal is cited, in accordance with accepted academic practice. No use, distribution or reproduction is permitted which does not comply with these terms. 